\title{
La place du langage dans les premières rencontres entre soignants et soignés
}

\section{The role of language in the first meetings between caregivers and patients}

\author{
Christophe Coupé ${ }^{1,2}$ et Magali Ollagnier-Beldame ${ }^{3}$ \\ ${ }^{1}$ Department of Linguistics, The University of Hong Kong, Pokfulam Road, Hong Kong \\ ${ }^{2}$ Laboratoire DDL UMR 5596 (CNRS, Université Lumière Lyon 2), France \\ ${ }^{3}$ Laboratoire ICAR UMR 5191 (CNRS, ENS de Lyon, Université Lumière Lyon 2), France
}

\begin{abstract}
Résumé. Le langage occupe une place importante dans le soin, qu'il accompagne et 'enveloppe' les différents actes des soignants ou qu'il soit le soin lui-même, en particulier dans un cadre psychothérapeutique. Nous insistons dans cet article sur le rôle du langage lors de la première rencontre entre un soignant et un soigné, en nous appuyant sur l'expérience vécue par le premier. Cette expérience est étudiée avec l'entretien microphénoménologique, une technique de collecte et d'analyse de données qui offre des descriptions précises, holistiques et en première personne d'une situation. Nous expliquons d'abord comment l'activité langagière s'inscrit dans le cadre d'une activité finalisée, pluridimensionnelle sur le plan expérientiel, qui vise à comprendre la problématique du soigné ainsi qu'à construire une relation avec lui. Nous nous penchons alors sur les différentes facettes du langage du patient auxquelles le soignant prête attention, puis décrivons le langage du soignant ce qu'il dit et se dit - à l'aide des actes de langage et de leur fonction illocutoire. Il apparait que le langage sert à bien davantage qu'échanger des informations, et occupe pour des soignants de professions diverses un rôle clé dans la dynamique micro-expérientielle, que ce soit pour comprendre l'autre, ajuster son comportement ou proposer un accompagnement.
\end{abstract}

\begin{abstract}
Language fulfils an important function in care, whether it accompanies and 'surrounds' caregivers' various acts or is care itself, in particular in a psychotherapeutic framework. We insist in this article on the role language plays during the first meeting between a caregiver and a patient. We rely on the caregivers' lived experience, as it can be studied with the microphenomenological interview - a process of data collection and data analysis which offers fine-grained, first-person descriptions of someone's holistic experience in a given situation. We first explain how linguistic interactions are part of a broader finalized activity, with various experiential dimensions, which aims to understand the problematic of the patient and to build a relationship with her/him. We then assess the different facets of the patient's language to which the caregiver pays attention, and describe the caregiver's language what s/he says to the other and to her/himself - with the help of speech acts and their illocutionary function. It appears that language is used for much more than exchanging information and, for caregivers of various professions, plays a key role in the micro-experiential dynamics, whether to understand the patient, adjust one's behavior or offer support.
\end{abstract}




\section{Introduction}

Dans de nombreux pays occidentaux, les soins sont si présents qu'ils en deviennent presque invisibles. En effet, les infirmiers, médecins, kinésithérapeutes, psychothérapeutes, etc. tissent un réseau très dense de compétences et de pratiques au service des personnes au quotidien. Une éthique des soins peut être promue (Tronto, 2009), qui insiste sur l'importance de prêter attention à autrui et d'ériger le soin et le bien-être en piliers de nos sociétés. Dans ce contexte, mieux comprendre ce qui se joue dans les pratiques ordinaires des soignants prend tout son sens. Que veut dire être soignant et prendre soin d'une autre personne ? Quelle en est l'expérience subjective et en quoi la pratique réelle diffère-t-elle de la pratique prescrite?

Les thématiques précédentes peuvent être abordées en particulier en se posant la question de la place du langage dans le soin. La communication occupe intuitivement une place importante, et le soignant et le soigné peuvent échanger sur de multiples facettes du soin : en quoi est-il nécessaire, comment est-il réalisé, quelles peuvent être ses conséquences, etc. Ces échanges peuvent permettre au soigné de mieux comprendre et accepter ce qui l'affecte, et de façon symétrique au soignant de faciliter des actes potentiellement difficiles à accepter (chimiothérapie, soins palliatifs, etc.). Cette communication peut également concerner des personnes proches du soigné et qui l'accompagnent dans sa prise en charge thérapeutique.

Nous nous proposons dans cet article de décrire de façon plus précise les interactions langagières précédentes, en nous centrant sur un épisode particulier du processus de soin : la première rencontre entre un soignant et un soigné. Notre approche s'ancre dans l'expérience subjective du soin et adopte le point de vue du soignant. Dans cette partie introductive, afin d'offrir un cadre général aux processus langagiers que nous étudierons ensuite, nous apportons quelques précisions sur la relation soignant - soigné, en particulier lors d'une première rencontre. Nous dressons également une rapide esquisse de la dimension expérientielle du soin pour le soignant, et soulignons l'intérêt d'une épistémologie en première personne.

\subsection{La relation soignant - soigné dans les premiers instants du soin}

Dans la plupart des situations de soins, établir et maintenir la relation intersubjective entre soignant et patient est un enjeu crucial pour la qualité de l'accompagnement. En contrepoint du modèle biomédical dominant jusque dans les années 1970, le modèle biopsychosocial développé en 1977 par Engel (Siksou, 2008) souligne que i) la relation médecin-patient influence le résultat médical, ne serait-ce qu'en influençant l'adhésion au traitement, ii) l'efficacité du médecin est liée au fait que sa personnalité est un instrument de changement thérapeutique, et iii) l'expérience subjective du patient mérite autant d'attention que sa maladie (ibid.). Engel relie ainsi les facteurs biologiques, psychologiques et sociaux en un système causal complexe, et contribue à contrer la déshumanisation de la médecine et l'infantilisation des patients. Cette perspective a récemment été renforcée, par exemple avec le développement de l'éducation thérapeutique des patients (Vargas-Schaffer et Cogan, 2014) et le rôle croissant des " patients experts » (Cuvelier, 2018). En psychothérapie, l'importance de la relation intersubjective a été montrée par des études sur l'« alliance thérapeutique » (Macewan, 2008), la «relation thérapeutique» (Ardito et Rabellino, 2011) et la « synchronisation affective » (Stern, 2004). Ces travaux étudient les moyens par lesquels un thérapeute et un patient s'engagent mutuellement pour conduire à un changement bénéfique pour le second. Barber et al. (2000) ont par exemple examiné si le résultat d'un traitement dépend d'une alliance thérapeutique positive à ses débuts. McAllister et al. (2004) ont également souligné la contribution des premiers rendez-vous à l'alliance thérapeutique et à la réussite du traitement. 
Malgré la considération croissante accordée aux patients par les soignants, le premier rendezvous est caractérisé par une asymétrie de rôle et de situation. Les deux personnes se voient attribuer des rôles spécifiques, pouvant façonner leur comportement : alors que le soignant est le dépositaire du savoir médical, occupant une place surplombante, le patient est généralement dépourvu de ce savoir et affaibli par ce qui l'amène. Cependant, ils sont tous deux davantage que ce que les règles sociales et la situation les contraignent à être. Ils conservent ce qu'ils sont en tant que personnes, ce qui contribue à une interaction plus symétrique. Ceci peut néanmoins rester en arrière-plan de leurs interactions car la formation des soignants insiste fortement sur le rôle qu'ils doivent adopter, notamment en laissant de côté leurs émotions face aux patients.

Evaluer l'influence de la personnalité et du rôle de chaque protagoniste est une question éthique importante des premiers rendez-vous de santé. Le soignant peut prêter attention à différentes caractéristiques de son patient, et bien sûr en particulier à la façon dont celui-ci s'exprime - des propriétés de la voix à ce qui est dit. Evaluer pour dresser un portrait juste peut par la suite favoriser une relation thérapeutique plus authentique et plus efficace. Cette tâche n'est toutefois pas aisée, d'abord parce que la rencontre avec de nouveaux patients est répétitive et habituelle. Bien que les premiers rendez-vous puissent être des moments remarquables pour un nouveau praticien, les habitudes s'installent rapidement et diminuent l'attention qu'il peut y porter. Deuxièmement, bien que répétitives, les premières rencontres engagent la part personnelle des soignants, qui peuvent éviter les comportements créant une gêne. Troisièmement, la majorité des soignants ne sont pas formés à prêter pleinement attention à leur vécu de ce moment crucial, au temps t ou plus tard de manière réflexive. Le moment de la rencontre constitue donc souvent un angle mort.

\subsection{La dimension expérientielle du processus de soin}

La grande majorité des soignants suit une formation initiale théorique et pratique, ainsi que des formations continues complémentaires. Le plus souvent, peu de place est offerte à l'expérience vécue dans les situations de soins, qu'il s'agisse de l'expérience du patient ou de celle du soignant. L'anthropologue Byron Good s'est intéressé à la manière dont les soignants tiennent compte (ou non) de l'expérience des patients. Son travail sur la « construction » des professionnels de santé montre comment les étudiants en médecine développent pendant leur cursus leurs attitudes et leur style d'écoute, notamment en ce qui concerne la voix des patients et les récits de maladie (Good, 1994). Good a étudié les qualités linguistiques de ces récits et a montré que le contexte de la narration influence la manière dont l'histoire est structurée. Ceci ouvre la voie à une analyse de la relation complexe entre l'expérience du patient et ses représentations narratives de la maladie.

Plus largement, la prise en compte de l'expérience vécue - du patient ou du soignant - offre des perspectives supplémentaires par rapport aux études qui considèrent seulement des données observables et/ou des rapports verbaux non expérientiels. Par exemple, OllagnierBeldame et Cazemajou (2019) ont mis en évidence un certain type de jugement apparemment, central dans les compétences intersubjectives des soignants mais peu étudié : les « jugements incarnés », appelés aussi « jugements intellectuels » par Burloud (1927). Il s'agit de jugements liés à une pensée intuitive et à un mode spécifique de conscience (Messer, 1906). Généralement exprimés par «je sens...» (par exemple «je sens quelque chose d'étrange », « je sens que quelque chose lui arrive »), ils ne transmettent pas seulement une sensation mais sont directement liés à l'activité intérieure non-conscientisée des soignants et à leurs compétences accumulées. De tels résultats illustrent comment l'activité de rencontre se met en place et se déploie dans le temps, et ouvrent la voie à la pratique réflexive des soignants. Cette dimension du soin est au cœur de l'analyse des pratiques (Henry, 2019), en tant que composante essentielle d'une éthique du soin où l'expérience subjective des soignants 
joue un rôle central. Ainsi, l'accès à l'expérience vécue, sa description précise et son analyse ont le potentiel de transformer les représentations et les attitudes des soignants envers leurs futurs patients. La section ci-dessous présente une épistémologie qui permet une telle étude de l'expérience subjective.

\subsection{Une épistémologie en première personne pour aborder l'expérience}

L'étude de l'expérience vécue requiert une épistémologie spécifique. Les approches phénoménologiques différencient les perspectives en première, deuxième et troisième personne en séparant la perspective du sujet qui vit l'expérience de la perspective d'un autre sujet, tel que le chercheur (Depraz et al., 2003). L'épistémologie en première personne considère la subjectivité telle qu'elle est vécue par le sujet lui-même, "de l'intérieur» (Depraz, 2014 ; Shear et Varela, 1999). Elle englobe à la fois les perspectives en première personne (le « je » qui parle est direct, immédiat) et en deuxième personne (le « je » qui parle est médiatisé par une autre personne, par exemple un chercheur).

L'épistémologie en première personne s'oppose à l'épistémologie en troisième personne selon laquelle la subjectivité et l'expérience vécue sont généralement considérées comme des épiphénomènes ou hors de portée de la science (Vermersch, 2000a), les comportements observables étant examinés selon des catégories prédéfinies. Ces considérations sont liées à une défiance concernant la validité des données tirées de l'introspection (Nisbett et Wilson, 1977), bien que plusieurs études aient redonné une légitimité à celles-ci (Hurlburt et Heavey, 2006 ; Petitmengin et al., 2013). L'épistémologie en première personne n'est cependant pas une épistémologie de l'immédiateté puisque l'expérience, bien que vécue et profondément intime, a une dimension pré-réfléchie. Y accéder en détail suppose une objectivation de sa subjectivité, ce qui n'est pas aisé malgré l'apparente transparence de l'intimité et de la familiarité. Ainsi, les perspectives en première et deuxième personne ne doivent pas être confondues avec la donation immédiate, c'est-à-dire, pour le sujet, une illumination soudaine, claire et distincte (Vermersch, 2000b).

Comme le souligne Pauen (2012 : 37-38), «le fait d'être épistémiquement lié à des faits concernant soi-même ( "je ») n'est pas une condition suffisante pour relever de la perspective en première personne: On peut aussi avoir un point de vue objectif, à la troisième personne, sur son mal de tête. [...] Ce qu'il faut, c'est une différence non pas en termes d'objet épistémique, mais plutôt en termes d'accès épistémique - même s'il peut s'avérer nécessaire de se référer à des objets épistémiques spécifiques afin de clarifier quel est le type d'accès spécifique. Le point décisif semble être qu'il existe certaines caractéristiques de soi qui requièrent un type spécifique d'accès épistémique " (notre traduction). Cette question cruciale de l'accès épistémique à l'expérience a conduit au développement de nombreuses méthodes de collecte de données expérientielles (Gendlin, 1997 ; Giorgi, 2009 ; Hurlburt et Heavey, 2006 ; Petitmengin, 2001 ; Vermersch, 1994), offrant différentes possibilités d'« enquête » et de description expérientielle, selon le type d'expérience et les objectifs de recherche. De nombreuses recherches mobilisent en particulier l'entretien microphénoménologique (Dieumegard et al., 2020 ; Ollagnier-Beldame et Cazemajou, 2019 ; Petitmengin, 2001 ; 2006 ; Przyrembel et Singer, 2018), y compris pour des situations de soins (Denis, 2016 ; Ollagnier-Beldame et Cazemajou, 2019). 


\section{Méthodologie}

\subsection{Un outil de collecte de données : l'entretien micro-phénoménologique}

Nous avons recueilli nos données avec l'entretien micro-phénoménologique, également appelé entretien d'explicitation. Cette méthode a été développée par Pierre Vermersch (1994, 2012), Claire Petitmengin (2006), Petitmengin et al. (2019) et les membres du GREX (Groupe de Recherche en EXplicitation). Contrairement aux autres types d'entretiens, centrés sur les représentations sociales (Moscovici, 2000), il s'intéresse à l'expérience vécue. Sur le plan théorique, il s'appuie sur des apports venant de la phénoménologie d'Edmund Husserl, la thérapie centrée sur le client de Carl Rogers, le focusing d'Eugene Gendlin, la théorie de la conscience de Jean Piaget et la théorie de la mémoire affective de Georges Gusdorf. C'est une technique d'introspection rétrospective guidée, ce qui signifie que l'intervieweur (communément appelé B), à l'aide de questions soigneusement choisies, aide et guide la personne interrogée (appelée A) à laisser revenir et à revisiter une expérience passée.

Le caractère non-inductif des questions est capital, car il permet de guider l'attention de l'interviewé sans orienter le contenu de ses réponses. Concrètement, lors de l'exploration d'une expérience, la question «que percevez-vous? " est souvent plus adaptée que la question «que voyez-vous? » car elle ne préjuge pas de la modalité perceptive dominante pour la personne interrogée. Plus généralement, les effets perlocutoires des interventions sont contrôlés autant que possible pour éviter l'induction de faux souvenirs (Schacter, 2001). Les interventions de l'enquêteur visent à induire et à maintenir l'introspection chez l'interviewé, grâce à un lâcher prise favorisant le ressouvenir de l'expérience passée, appelé évocation. L'état de conscience légèrement modifié, qui favorise une présence à soi et un contact intime avec la situation passée, est appelé " position de parole incarnée ». Cette dernière n'est pas spontanée et peut s'arrêter si les récapitulations de l'intervieweur s'écartent trop des descriptions précises de l'interviewé. Une écoute attentive est donc essentielle, ainsi que des évaluations régulières à l'aide d'indices verbaux et non-verbaux complémentaires - regard non focalisé, ralentissement du débit de la parole, utilisation du pronom personnel «je » plutôt que «nous » ou « on », etc. Globalement, les interventions sont structurées par i) les objectifs de l'entretien, c'est-à-dire les informations que l'on souhaite recueillir, ii) ce que l'on sait théoriquement de l'expérience, et qui correspond à des processus génériques d'assez haut niveau (cf. Figure 1 ci-dessous), et iii) ce que dit l'interviewé.

L'objectif de l'entretien micro-phénoménologique est de recueillir des descriptions détaillées d'une expérience vécue singulière. Ces descriptions sont holistiques dans le sens où les différentes composantes de l'expérience (opérations cognitives, actions physiques, sensations, émotions, etc.) sont toutes considérées et jugées pertinentes. Ce que l'on recherche, ce sont des descriptions spécifiques de ce qui a été vécu, et non des généralisations dérivées de situations et d'habitudes répétées, ni des opinions. Ceci explique en particulier pourquoi les questions " pourquoi... ? » sont écartées. Les intervieweurs recherchent à la fois la fragmentation de l'expérience, qui correspond à la succession diachronique de microévénements expérientiels composant les expériences à plus grande échelle, et l'expansion qualitative, qui permet de décrire les qualités expérientielles à un moment donné. Les aspects pré-réfléchis de l'expérience étudiée, c'est-à-dire la partie de celle-ci restée sous le seuil de la conscience réflexive, font l'objet d'une attention particulière car ils contiennent des informations utiles sur ce qui a été vécu et son déroulement. Ceci inclut notamment la source possible des erreurs commises au cours d'une activité ou les aspects implicites des connaissances expertes. 


\subsection{Collecte et traitement des données}

Nous avons interrogé 13 soignants de diverses professions entre janvier 2015 et décembre 2016: 2 orthophonistes (femmes), 2 médecins généralistes (femme/homme), 1 dentiste (homme), 1 kinésithérapeute (femme), 3 psychothérapeutes (femmes/homme), 4 infirmiers avec diverses spécialisations (femmes/homme). Tous les participants étaient de langue maternelle française. Chaque entretien ${ }^{1}$ a duré entre 45 et 90 minutes $^{2} .11$ des 13 enregistrements ont été entièrement transcrits ${ }^{3}$ et 7 ont été retenus pour une analyse plus approfondie.

Notre méthode d'analyse est qualitative et fait face aux défis épistémologiques et méthodologiques de toutes les approches qualitatives (Saldaña, 2011). Elle repose sur une approche itérative éprouvée, afin d'extraire des catégories descriptives significatives de l'expérience. À la suite de Vermersch (2012), Petitmengin (2006) et ValenzuelaMoguillansky et Vásquez-Rosati (2019) ont affiné la méthode d'analyse des entretiens microphénoménologiques pour les besoins de la recherche. Vermersch a proposé un processus de sémiose itérative pour transformer progressivement l'expérience de la personne interrogée en connaissances. Ce processus commence par l'expérience elle-même, et la façon dont elle est mise en mots par la personne interrogée. À ce stade déjà, une transformation sémiotique se produit, car certaines expériences sont plus difficiles que d'autres à mettre en mots. L'étape suivante est la transcription des entretiens enregistrés, pendant laquelle divers aspects du discours (intensité, rythme) peuvent être laissés de côté par souci de simplicité, bien qu'il soit possible d'y revenir ultérieurement si besoin.

Nous avons traité nos transcriptions selon les étapes couramment suivies par les chercheurs en micro-phénoménologie Les premières consistent en une clarification et une simplification du matériel transcrit pour accroitre sa lisibilité et mettre en valeur le contenu signifiant, c'està-dire les descriptions expérientielles. Les étapes suivantes visent à dégager les moments clés de l'expérience et une vue d'ensemble, et à réorganiser le contenu pour passer de la chronologie de l'entretien à la chronologie de l'expérience. Le résultat, appelé déroulement temporel de l'expérience, constitue la base des analyses ultérieures. Dans le cas de notre étude, certaines actions du patient, telles que perçues et rapportées par le soignant, ont été conservées pour faciliter la compréhension de la situation et de sa dynamique.

Une fois le déroulement temporel de l'expérience passée reconstruit, le chercheur a accès à une description riche et condensée de l'expérience, avec l'articulation de microphénomènes expérientiels successifs composés de perceptions et de sensations, d'actions, de prises de décision, etc.

Au-delà du processus d'extraction précédent, Vermersch a également proposé un modèle de l'action (Faingold, 1997 ; Vermersch, 1994), qui articule plusieurs phases successives au

1 Avant l'entretien micro-phénoménologique proprement dit, les personnes interrogées ont été pleinement informées des objectifs, des méthodes et du cadre institutionnel du projet de recherche. L'accord explicite pour l'enregistrement de l'entretien a été obtenu oralement, en mentionnant clairement l'anonymat et l'analyse ultérieure des données. Des informations sur le niveau de formation, le nombre d'années de pratique et le contexte professionnel d'intervention (pratique privée/publique, patients, etc.) ont également été recueillies. Aucune information permettant une quelconque identification des patients mentionnés pendant les entretiens n'a été recueillie.

2 Durée totale des enregistrements: $14 \mathrm{~h} 7 \mathrm{~m} \mathrm{42s}$. Durée des données micro-phénoménologiques

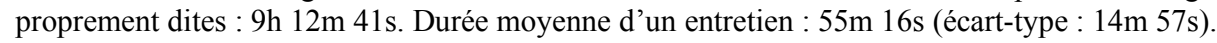

${ }^{3}$ Nombre total de mots pour les transcriptions (après ajout d'informations temporelles et numérotation des interventions pour l'enquêteur et l'interviewé): 117 142. Nombre moyen de mots par transcription : 11714 (écart-type : 3 286). Les transcriptions sont disponibles sur demande auprès des auteurs. 
cours de l'action. Il est utile comme support pour guider la personne interviewée à décrire chronologiquement son expérience. Selon lui, toute action s'organise chronologiquement en 1) une prise d'informations, puis 2) l'identification des informations pertinentes, 3) une prise de décision et 4) l'exécution/la mise en œuvre de ce qui a été décidé. Les personnes interrogées font généralement état des étapes 2) et 4) de manière plus spontanée, mais peuvent aussi être interrogées explicitement sur les autres étapes.

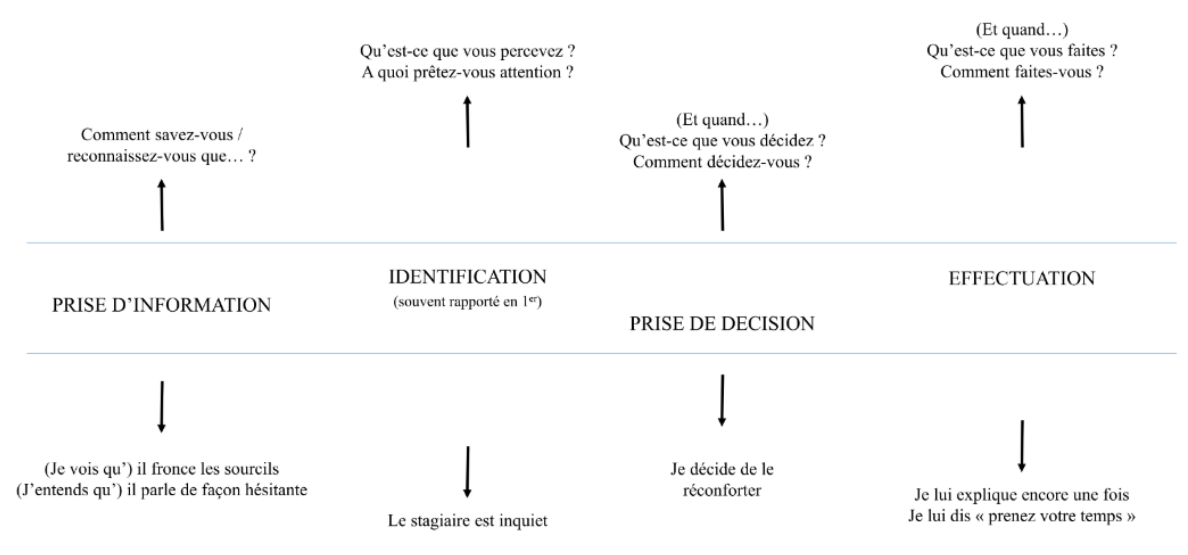

Figure 1. Le modèle de l'action avec les questions et les réponses possibles dans un entretien microphénoménologique.

Dans la section suivante, nous rapportons les résultats de l'approche analytique précédente pour nos données. Ceci nous permet de construire un cadre pour aborder ensuite, dans la section 4 , le rôle du langage lors d'une première rencontre entre soignant et soigné.

\section{Dimensions de l'expérience du soignant lors d'une première rencontre avec un patient}

\subsection{Synchronie et diachronie des actes}

Comment caractériser de façon fine l'expérience de rencontrer un patient pour la première fois ? Nous avons analysé les microcomposants de cette expérience tels qu'ils apparaissaient dans nos entretiens. Notre but premier était de façonner un ensemble de dimensions de l'expérience et, à l'intérieur de chacune d'entre elles, un ensemble de catégories reflétant la diversité des situations et des actions réalisées. Notre position est en effet que caractériser le panorama des possibles est un premier pas vers une compréhension plus profonde puisque sans description précise et fidèle, un phénomène ne peut être correctement étudié.

Nous avons dénommé les microcomposants de l'expérience phénomènes micro-expérientiels (PME), et avons considéré les deux axes centraux de la micro-phénoménologie: la succession de ces PME au cours du temps, c'est-à-dire leur diachronie, et leurs différentes dimensions à un instant donné, c'est-à-dire leur synchronie. Pour ce qui est de la diachronie, nous sommes partis du modèle de l'action et de ses quatre étapes reliées causalement : i) la prise d'information, ii) l'identification de la situation (sur la base de l'information collectée), iii) la prise de décision (après identification des enjeux de la situation) et iv) l'effectuation. Nous avons trouvé que la troisième étape était rarement identifiable en tant que telle, et était en fait plutôt déduite à rebours à partir de la dernière étape. Ceci nous a amené à considérer seulement trois catégories principales d'actes - au sens large du terme, c'est-à-dire en 
incluant les actes perceptifs, cognitifs, langagiers, moteurs, etc. - pour le soignant. Nous avons remplacé le terme d'identification par celui de jugement, plus fidèle au contenu de nos entretiens, et conservé le terme d'effectuation mais y en incluant de façon implicite la prise de décision.

La considération des différentes facettes synchroniques des PME nous a conduit à distinguer un ensemble de «portées » pour les actes. Celles-ci reflètent la situation du soin et de ses acteurs principaux - le soignant et le soigné - mais également la perspective du soignant sur la situation. Pour la prise d'information, il est apparu tout d'abord que le soignant peut prendre de l'information de façon externe, i.e. en dehors de son corps, mais aussi de façon interne, en lui-même. Même si intuitivement l'information externe semble associée au soigné et l'information interne au soignant, la seconde peut en fait aussi être à propos d'autrui. D'une façon similaire, les jugements peuvent porter sur le soignant ou le soigné, mais également sur leur relation ou la situation. Enfin, les actions effectuées peuvent affecter les deux parties de la relation de soins, avec des régulations et des interventions de la part du soignant. Le tableau 1 résume et illustre le champ des possibles observés dans nos entretiens.

Tableau 1. Catégories et portées des actes réalisés par le soignant telles qu'extraites du verbatim des entretiens, avec illustrations.

\begin{tabular}{|c|c|c|}
\hline $\begin{array}{l}\text { Catégorie } \\
\text { d'actes }\end{array}$ & Portée des actes & Exemple de verbatim \\
\hline \multirow{3}{*}{$\begin{array}{c}\text { Prise } \\
\text { d'information }\end{array}$} & $\begin{array}{l}\text { Information } \\
\text { externe sur l'autre }\end{array}$ & $\begin{array}{l}\text { "j'vois quelqu'un un peu effrayé qui repose } \\
\text { précipitamment un magazine une bd ou une revue } \\
\text { sur le porte-revues sur la table basse qui attrape } \\
\text { son manteau» }\end{array}$ \\
\hline & $\begin{array}{l}\text { Information } \\
\text { interne sur soi }\end{array}$ & "y a comme une chaleur dans un coin de ma tête" \\
\hline & $\begin{array}{l}\text { Information } \\
\text { interne sur l'autre }\end{array}$ & $\begin{array}{l}\text { "il y a une résonance c'est dans mon corps je sens } \\
\text { qu'il y a un vide là où est l'autre " }\end{array}$ \\
\hline \multirow{4}{*}{ Jugement } & Soi & $\begin{array}{l}\text { "j'me dis que j'suis pas la personne que les gens } \\
\text { pensent que j'suis à ce moment-là » }\end{array}$ \\
\hline & L'autre & $\begin{array}{l}\text { "je trouve que le père et le fils se ressemblent } \\
\text { beaucoup (rires)" }\end{array}$ \\
\hline & La relation & $\begin{array}{l}\text { "je me dis tiens là il va y avoir quelque chose de } \\
\text { dynamique je sens que l'entretien va être } \\
\text { dynamique (...) c'est vécu par tout le corps" }\end{array}$ \\
\hline & La situation & $\begin{array}{l}\text { "je me dis que ça va être encore une histoire } \\
\text { difficile à entendre" }\end{array}$ \\
\hline \multirow{3}{*}{ Effectuation } & Réguler (sur soi) & $\begin{array}{l}\text { "j'essaie de garder cette attitude rassurante à son } \\
\text { égard en me disant que c'est aussi comme ça que } \\
\text { ce qui va suivre sera de bonne qualité " }\end{array}$ \\
\hline & $\begin{array}{l}\text { Intervenir (sur } \\
\text { l'autre) }\end{array}$ & $\begin{array}{l}\text { "avec le regard je vais chercher ce qu'il amène lui } \\
\text { à ce moment-là » }\end{array}$ \\
\hline & Autres & " je me mets aussi à me tortiller » \\
\hline
\end{tabular}




\subsection{Modalités expérientielles}

Outre les actes et leurs portées, nous avons conclu au besoin de spécifier les principales modalités expérientielles liées à la prise d'information, au jugement ou à la réalisation d'actions. Ces modalités recoupent nos cinq sens, mais certains étaient absents quand d'autres conditions demandaient à être prises en considération. Nous avons ainsi considéré :

- Les modalités visuelle, auditive et tactile ${ }^{4}$;

- Les sensations, qui correspondent à des perceptions internes pouvant être qualifiées d'intéroceptives (en opposition à extéroceptives, comme les cinq sens) ;

- La modalité extra-sensorielle ${ }^{5}$;

- Le langage interne, i.e. les productions linguistiques dirigées vers soi ;

- Le langage (externe), dirigé vers les autres ;

- L'activité motrice ;

- Les jugements incarnés, présentés en section 1.2, c'est-à-dire des impressions non analysées qui deviennent des idées, des jugements et des raisonnements. Ces jugements conclusifs, experts et intuitifs, émergent habituellement après une série de prises d'informations et de jugements, sous la forme d'une prise de conscience.

Par exemple, les informations prises à l'extérieur du corps peuvent être de nature visuelle ou auditive, les informations internes peuvent consister en sensations, les effectuations en actions physiques, en discours adressé au patient ou encore en langage interne adressé à soimême. Le tableau 2 offre des exemples pour ces différentes modalités expérientielles.

Tableau 2. Modalités expérientielles pour le soignant, avec illustrations.

\begin{tabular}{|c|c|}
\hline Modalité expérientielle & Exemple de verbatim \\
\hline Vision & $\begin{array}{l}\text { "il avait le regard assez réveillé des yeux clairs assez lumineux } \\
\text { il n'a pas le regard éteint" }\end{array}$ \\
\hline Audition & $\begin{array}{l}\text { "j'ai perçu à ce moment-là qu'il a un gros trouble } \\
\text { d'articulation " }\end{array}$ \\
\hline Toucher & "je prête attention (...) à la poignée de main en fait » \\
\hline Sensation & $\begin{array}{l}\text { "je sens que il y a une détente des bras et là je sens même qu'il } \\
\text { y a une détente au niveau des épaules une ouverture près des } \\
\text { clavicules " }\end{array}$ \\
\hline $\begin{array}{l}\text { Modalité } \\
\text { extra-sensorielle }\end{array}$ & $\begin{array}{l}\text { "il n'existe pas (...) si j'essayais de le toucher il n'y aurait pas } \\
\text { de texture ça s'effondrerait ou ça serait un fantôme je passerais } \\
\text { à travers c'est une sensation que l'autre n'a pas de substance » }\end{array}$ \\
\hline Langage interne & "je me dis tiens là il va y avoir quelque chose de dynamique» \\
\hline Langage (externe) & $\begin{array}{l}\text { "je dis ok moi je suis prête pour travailler avec vous sur le } \\
\text { viol»" }\end{array}$ \\
\hline Activité motrice & "c'est moi qui ai ouvert la porte » \\
\hline Jugement incarné & $\begin{array}{l}\text { "il y a cette idée un petit peu globale de ah tiens ils présentent } \\
\text { bien tous les deux»" }\end{array}$ \\
\hline
\end{tabular}

${ }^{4}$ Notre corpus ne contenait pas d'instances pour le goût ou l'odorat.

5 Différente d'une perception intéroceptive ou extéroceptive, une modalité extra-sensorielle est exprimée par le sujet avec des notions de texture, de substance, de forme, etc. 


\subsection{Activité finalisée}

Comme la plupart des professionnels, le soignant réalise une activité dite finalisée (Daniellou et Rabardel, 2005). Une activité finalisée est « une activité centrée sur l'accomplissement d'objectifs pratiques » (Garfinkel, 1967) (notre traduction), qui diffère d'une activité « ouverte » pour laquelle les résultats attendus ne sont pas définis ou seulement de façon imprécise. Pour les soignants, parmi les buts à accomplir se trouvent le besoin d'établir un diagnostic et celui de construire une alliance thérapeutique (Rogers, 1957). Nous avons questionné nos interviewés et ceux-ci ont en effet décrit des actions concourant à l'établissement d'un diagnostic, par exemple « je me dis à ce moment-là tiens on va aller creuser peut-être que c'est pas un gamin asperger peut-être qu'il est haut potentiel » (un orthophoniste) ou « il y a vraiment cette casquette thérapeutique qui est là qui est vraiment présente et qui bon (inspire profondément) sait » (un psychothérapeute). Ils ont également pointé des actes qui participent à l'élaboration d'une alliance thérapeutique, par exemple "je lui demande de me dire qui vit avec eux à la maison... Une fois qu'il m'a cité toutes les personnes qui y avait dans la famille je lui demande si y a des poissons rouges des tortues des hamsters (...) le plus souvent ça les fait rire » (une infirmière). Nous trouvons dans nos données de nombreux verbatim liés à la prise d'information, ce qui nous confirme que celleci est au cœur de l'activité des soignants. Par exemple, un orthophoniste nous a dit "je me dis tiens là il va y avoir quelque chose de dynamique je sens que l'entretien va être dynamique (et quand je me dis "ça va être un moment dynamique") j'entends ça en même temps je me le dis et en même temps je sens que c'est vécu par tout le corps ».

L'activité finalisée du soignant est à la fois productive et constructive : productive car centrée sur l'accomplissement d'un projet et prenant en compte les caractéristiques de la situation, et constructive car entrainant le développement de ressources internes et externes, qu'il s'agisse d'instruments, de compétences, de concepts ou encore de systèmes de valeur (Rabardel, 2005). La dimension productive transparait souvent dans les entretiens, par exemple lorsqu'un dentiste dit «je retiens ma respiration j'respire avec le haut du corps... je me prépare à ce que tout soit plus long avec cette personne ». Quant à la dimension constructive, elle est explicite quand une médecin rapporte par exemple « c'est là que du coup je commence à regarder un petit peu la sémiologie (...) la lenteur du déplacement, la difficulté à exécuter les mouvements (...) ça m'a fait penser aux différents patients que j'ai pu voir par exemple, qui étaient atteints de la maladie de Parkinson (...) ça a comme été une compilation de patients qui avaient le même mode de déplacement (...) une somme de de cas cliniques qu'on avait acquis en nous ». Le soignant vise à accompagner son patient selon sa situation et sa problématique, et peut s'appuyer sur des acquis passés alors même qu'il en génère de nouveaux.

\subsection{Un cadre spécifique pour l'activité langagière}

Les trois sections précédentes permettent de brosser le cadre, expérientiel, du rôle du langage dans l'activité de soin. À un niveau microscopique tout d'abord - niveau qui rejoint le modèle de l'action de la micro-phénoménologie -, ce rôle prend place au sein d'un processus expérientiel multidimensionnel et organisé temporellement en série de micro-schémas impliquant la prise d'information, le jugement et l'effectuation. Si le langage correspond à la fois à une modalité expérientielle et à une panoplie d'actions du soignant, il n'est cependant qu'une possibilité parmi de nombreuses autres, et doit se comprendre en articulation avec elles. À un niveau plus mésoscopique, le rôle du langage s'inscrit dans une activité finalisée, dirigée en particulier vers un diagnostic et l'établissement d'une alliance thérapeutique, qui s'appuie sur un ensemble de compétences et peut également générer de nouveaux acquis. La synthèse de ces deux niveaux de description de l'activité permet de comprendre comment 
des microphénomènes expérientiels qui impliquent différents processus langagiers peuvent par conjugaison permettre d'atteindre des objectifs à moyen terme. C'est cette articulation que nous examinons dans la prochaine section.

\section{Place du langage dans l'expérience du soignant}

\subsection{Prêter attention au langage de l'autre}

Un recensement des différents objets d'attention du soignant, alors qu'il rencontre un nouveau patient, indique clairement qu'il considère un ensemble d'éléments langagiers. Il est possible de regrouper ces éléments en plusieurs catégories complémentaires, qui soulignent l'intérêt porté tant à la forme qu'au fond.

Une première catégorie correspond aux noms et prénoms du patient, c'est-à-dire à des marqueurs sociolinguistiques de son identité. Elle diffère des catégories suivantes en ce qu'elle semble moins porteuse de sens et se rattache aux tous premiers instants de la relation. Le nom et le prénom du patient sont en effet souvent le tout premier « contact » qu'a avec lui le soignant. L'enjeu est d'établir un premier lien.

Une seconde catégorie porte sur les caractéristiques de la voix du patient : sa hauteur, sa vitesse d'élocution, ses modulations, ses possibles troubles, etc. Il s'agit d'une certaine façon de la « surface » du langage, de la forme primairement incarnée et physiologique de celui-ci. Cette surface langagière entre en interaction avec un ensemble d'autres caractéristiques du patient, eux aussi objets d'attention de la part des soignants, à savoir la respiration, la tension physique, les possibles silences, les gestes intentionnels ou non, etc.

Une troisième catégorie s'inscrit à mi-chemin entre la surface et le fond. Elle contient des caractéristiques de la langue employée sans pour autant se centrer sur le contenu exact de ce qui est dit : sa cohérence, son ou ses registre(s), sa sophistication, son caractère loquace ou non, ou encore la présence de certaines expressions. Un élément en miroir, que nous pourrions par jeu de symétrie inclure dans cette catégorie, concerne la capacité d'écoute du patient, à laquelle certains de nos soignants ont rapporté prêter attention.

Une quatrième et dernière catégorie - la plus vaste - englobe ce que veut exprimer le patient, qu'il s'agisse du motif de sa consultation, de son histoire personnelle, de sa situation actuelle, etc. Il s'agit du sens que ce patient entend véhiculer par ses paroles, tel qu'il est perçu tout du moins par le soignant.

Le tableau 3 contient un ensemble d'illustrations pour les éléments précédents.

Tableau 3. Objets d'attention du soignant liés au langage, avec illustrations.

\begin{tabular}{|ll|}
\hline $\begin{array}{l}\text { Objet } \\
\text { d'attention }\end{array}$ & Exemple de verbatim et profession du soignant \\
\hline $\begin{array}{l}\text { Nom et } \\
\text { prénom }\end{array}$ & $\begin{array}{l}\text { "j'vois que y'a quelqu'un que j'connais pas c'est marqué "NP" comme } \\
\text { nouveautient donc cette dame c'que j'vois en premier c'est le nom " } \\
\text { (dentiste) }\end{array}$ \\
$\begin{array}{l}\text { "sa voix une voix blanche pas incarnée un peu vide qui peut comme un } \\
\text { peu dérailler en fonction de ce qu'elle dit » (psychologue) } \\
\text { "j'ai perçu à ce moment-là qu'il a un gros trouble d'articulation qui le } \\
\text { gêne au quotidien » (orthophoniste) }\end{array}$ \\
\hline
\end{tabular}




\begin{tabular}{|c|c|}
\hline \multirow{2}{*}{ Langue } & $\begin{array}{l}\text { "quand elle parle d'elle y a quelque chose qui se fige y a quelque chose } \\
\text { qui est pas fluide » (psychologue) }\end{array}$ \\
\hline & $\begin{array}{l}\text { "(la maman) qui parlait très bien donc je me rends compte rapidement } \\
\text { que c'est pas une maman qui vient d'arriver en France " (infirmière psy) }\end{array}$ \\
\hline Contenu de & $\begin{array}{l}\text { "elle me dit tout ce qui va pas qu'il tape les autres enfants qu'il est } \\
\text { insupportable qu'il respecte pas les règles " (infirmière psy) }\end{array}$ \\
\hline $\begin{array}{l}\text { ce qui dit le } \\
\text { patient }\end{array}$ & $\begin{array}{l}\text { "il dit que normalement il parle pas facilement qu'il a toujours peur de } \\
\text { pas réussir à parler et que là en fait il est en train de dire pas mal de } \\
\text { choses » (psychothérapeute) }\end{array}$ \\
\hline
\end{tabular}

Un premier point qui se dégage ainsi aisément est que le sens peut s'établir à différents niveaux de l'activité linguistique du patient, et que différents soignants peuvent a priori tirer parti de différentes façons du langage de l'autre. On peut remarquer ici qu'il est évident qu'un orthophoniste prête tout particulièrement attention à la voix d'un patient. Qu'un psychothérapeute soit à l'écoute de la cohérence du discours l'est tout autant. Le langage de l'autre sert la poursuite de leurs objectifs, objectifs distincts, puisque liés à des professions de soin différentes, mais similaires si l'on considère les finalités diagnostiques et d'alliance thérapeutique. Il apparait toutefois que les soignants interviewés - médecin, dentiste, orthophoniste, etc. - semblent tous enclins à une écoute à différents niveaux de l'activité langagière de leurs patients, et peuvent attribuer une signification à ces objets d'attention. En d'autres termes, prêter oreille aux caractéristiques de la voix n'est pas l'apanage des orthophonistes, pas plus que les caractéristiques de la langue utilisée ne sont la chasse gardée des psychothérapeutes. L'étude fine de la subjectivité des soignants révèle ainsi que les expériences du langage de l'autre sont peut-être plus productives que l'on pourrait le penser a priori, même si ce qu'interprète chaque soignant dépend du type de soin qu'il peut apporter. Dans certains cas, un rapport direct peut être établi entre ce dernier et l'attention portée à un aspect langagier spécifique ; c'est le cas des exemples ci-dessus. Dans d'autres cas toutefois, le lien est plus ténu et moins intentionnel, et l'impact sur le soin peut être variable, de très modeste à plus significatif.

Une autre remarque, d'ordre sémiotique, est que le langage est principalement envisagé comme un index / indice - au sens de Peirce - de la vie intérieure du patient. Ceci signifie qu'il est la manifestation causale de cette dernière, et dès lors une porte pour accéder à ce qui n'est pas visible: les ressources et capacités du patient, ses états internes (émotions, sensations, pensée, disposition mentale), son état psychique (lié à une possible pathologie), etc. Pour un psychothérapeute par exemple, le motif explicite de consultation peut ne pas correspondre exactement à ce qui motive de façon profonde le besoin de soin. Ce motif, combiné à d'autres observations, peut néanmoins jouer le rôle d'indice de ce qui amène réellement le patient et qu'il ne parvient pas à formuler. Le cas des orthophonistes est ici singulier, puisque le langage lui-même est pour eux l'objet premier du soin. Globalement, le rôle « indiciel » du langage ressort clairement de ce que nous ont rapporté les soignants. Par exemple, une orthophoniste rapporte que «je savais que c'était un patient qui venait pour la voix envoyé par sa phoniatre j'ai en tête que ce genre de patients ils viennent préoccupés pour eux, ils peuvent venir un peu chargés ». Un psychologue nous rapporte quant à lui que " quand elle le prononce (le terme) on voit bien qu'elle n'est pas ancrée dans le moment où elle le dit », ou encore, ce qui illustre les liens tissés entre objets d'attention langagiers et non-langagiers : " (elle est) en train de vivre dans son corps ce qu'elle est en train de me dire aussi... parce que elle bouge pas pareil quand elle m'écoute... et quand elle parle de ses souffrances... son corps incarne la souffrance dont elle parle ».

L'expérience du langage de l'autre présente donc plusieurs facettes, qui se retrouvent de façon répétée dans les phénomènes micro-expérientiels des soignants, et peuvent les guider dans la réalisation de leurs objectifs à un niveau plus macroscopique. 


\subsection{Les langages interne et externe du soignant}

Le langage du soigné et ce qu'en fait le soignant ne constituent pas la seule perspective sur le langage dans le soin. Le langage du soignant peut également être étudié, et une distinction intéressante se dessine entre ce qu'il adresse au patient (le langage externe) et ce qu'il s'adresse à lui-même. La frontière est ici entre un langage visible et un langage invisible, et le contraste est rendu possible par la nature subjective de nos données, qui nous donnent accès, au moins en partie, à l'univers privé du soignant.

Si l'on considère les catégories d'actes des soignants présentées en section 3.1, le langage du soigné se rapporte avant tout à la prise d'information et aux jugements qui en découlent. Le langage du soignant, quant à lui, est naturellement en lien avec ses jugements et ce qui est effectué. Plus précisément pour les effectuations, la dichotomie est de façon presque totale la suivante : le langage externe, adressé au patient, correspond aux interventions du soignant, alors que le langage interne correspond aux régulations qu'il exerce sur lui-même. Comment investiguer et en particulier comparer ces deux domaines ? Une option consiste à considérer les différents actes de langage réalisés.

Le langage sert à bien plus qu'à véhiculer de l'information, et les actes de langage pointent en direction de ce qu'il est possible de faire quand on dit, pour reprendre le titre de la traduction française d'un ouvrage bien connu de John Austin (1991). Dans la théorie d'Austin, les actes de langage présentent trois fonctions, à savoir les fonctions locutoires, illocutoires et perlocutoires. La fonction locutoire correspond à la production et l'émission du message même. La fonction illocutoire dépasse d'une certaine façon ce message et son sens immédiat pour référer au but recherché par l'émetteur. La fonction perlocutoire renvoie à l'effet que le message a sur celui qui le reçoit. Si l'on considère par exemple la question «tu as vu l'heure? » adressée à un ami lors d'un dîner à une heure avancée, la fonction illocutoire peut être de notifier à cet ami qu'il est tard, et la fonction perlocutoire la réaction de ce dernier de proposer de rentrer.

Les actes illocutoires sont traditionnellement envisagés pour des messages adressés à autrui (acte de langage en français vient d'ailleurs de speech act en anglais). Il est toutefois raisonnable de les étendre à un discours adressé à soi-même, dont la fonction locutoire est de nature différente - elle ne nécessite pas l'émission de sons, quand bien même on peut articuler à voix haute ou basse ce que l'on s'adresse - mais qui comporte lui aussi des objectifs.

L'examen de nos entretiens révèle un ensemble d'interventions et de régulations. Pour ce qui est des premières, le soignant peut chercher à accueillir, saluer, inviter à entrer, informer, expliquer, répondre mais aussi demander / questionner, proposer, interrompre ou encore rassurer. En ce qui concerne les secondes, le soignant peut chercher à se rassurer, se protéger, s'ancrer, accueillir intérieurement, "coiffer sa casquette de thérapeute ", s'autoriser à commettre une erreur, se blâmer, changer son « énergie » ou " se nettoyer » mentalement, rester ouvert et curieux, se disposer à quelque chose ou encore se contenir. Une partie de ces actes n'impliquent pas une activité langagière, interne ou externe, ou tout du moins ne sont pas rapportés comme tels. Nos interviewés spécifient cependant d'autres actes dans leur dimension langagière, et il est alors légitime de considérer une fonction illocutoire. Le tableau 4 présente des illustrations de ces actes de langage et des effets recherchés. Il inclut également des exemples de jugements - de soi, de l'autre, de la relation ou de la situation - qui impliquent un discours adressé à soi-même et qui n'entrent pas dans la catégorie des régulations, quand bien même certains cas sont tangents. Nous pouvons souligner le cas intéressant dans lequel ce qui est adressé au patient a pour fonction de se rassurer soi-même : « moi j’ai besoin de faire cette blague pour meubler un petit peu aussi ». 
Tableau 4. Exemples illustrés de fonctions perlocutoires des actes de langage du soignant lors de ses interventions et régulations.

\begin{tabular}{|c|c|}
\hline Fonction illocutoire & Exemple de verbatim \\
\hline \multicolumn{2}{|l|}{ Intervention } \\
\hline Saluer & $\begin{array}{l}\text { "un bonjour assez franc vigoureux avec un volume haut" } \\
\text { "avec les petits je me baisse je me mets à leur niveau et je leur } \\
\text { dis bonjour" }\end{array}$ \\
\hline Inviter à entrer & "j'lui dis on va s'installer à côté » \\
\hline Informer & "je lui dis qu'il me reste pas beaucoup de temps" \\
\hline Expliquer & $\begin{array}{l}\text { "je leur demande de regarder autour d'eux... de vraiment } \\
\text { s'approprier l'environnement et je leur explique c'est un nouvel } \\
\text { environnement vous ne le connaissez pas je leur dis on a notre } \\
\text { cerveau reptilien lui il a besoin de savoir » }\end{array}$ \\
\hline Demander & " je lui demande si il sait pourquoi il vient me voir" \\
\hline Proposer & $\begin{array}{l}\text { "je propose à Antoine et à sa maman de se mettre sur les } \\
\text { fauteuils en face de moi... et au petit de mettre sur la table » }\end{array}$ \\
\hline Interrompre & "j’interromps ce dialogue» \\
\hline Rassurer & $\begin{array}{l}\text { "j'essaie de le rassurer je dis écoutez vous attendez X c'est } \\
\text { quelqu'un qui normalement est toujours à l'heure à ses rendez- } \\
\text { vous" }\end{array}$ \\
\hline Jugement & $\begin{array}{l}\text { "c'est quelqu'un qui a l'habitude des premiers contacts je me dis } \\
\text { il est en lien avec le monde qui l'entoure" } \\
\text { "je me dis elle fait n'importe quoi cette femme je me dis mais } \\
\text { comment c'est possible de faire vivre des trucs pareils à des } \\
\text { enfants" }\end{array}$ \\
\hline \multicolumn{2}{|l|}{ Régulation } \\
\hline Se rassurer & $\begin{array}{l}\text { "je me dis ça va aller je connais ça pas de soucis je connais ça } \\
\text { va ça m'inquiète pas » } \\
\text { "moi j'ai besoin de faire cette blague pour meubler un petit peu } \\
\text { aussi » }\end{array}$ \\
\hline Se blâmer & " je me dis franchement j'aurais pu préparer la pièce avant » \\
\hline $\begin{array}{l}\text { Accueillir } \\
\text { intérieurement }\end{array}$ & $\begin{array}{l}\text { "(je me dis) tu vas voir tu vas voir comment ça va se passer } \\
\text { accueille prends ce qui est là » }\end{array}$ \\
\hline $\begin{array}{l}\text { Changer son } \\
\text { énergie }\end{array}$ & $\begin{array}{l}\text { "je me dis bon on va accueillir on va voir ce qu'on peut faire } \\
\text { c'est changer d'énergie " }\end{array}$ \\
\hline Rester ouvert & $\begin{array}{l}\text { "et dire bon laissons ouvert accueillons ce qu'il se passe et } \\
\text { voyons ce qu'on peut faire avec" }\end{array}$ \\
\hline Se disposer à qqc & $\begin{array}{l}\text { " juste avant (que je la rencontre) je m'aiguisais je m'dis sois prêt } \\
c^{\prime} \text { est un moment important » }\end{array}$ \\
\hline
\end{tabular}


Il apparaît ainsi clairement que le langage sert à bien plus qu'échanger des informations entre soignant et soigné. Les fonctions illocutoires sont variées, tant pour ce qui est des interventions que des régulations, et soulignent la place prépondérante du langage dans le soin.

Les multiples instances d'actes de langage visant à rassurer l'autre ou à se rassurer soulignent que le soin est bien sûr en direction du patient, mais également, de façon interne, en direction du soignant. Cette remarque pointe en direction d'une problématique générale - le besoin social d'accompagner et valoriser les soignants - mais permet de constater de façon explicite que les soignants peuvent, les premiers, de façon consciente, remplir ces tâches à leur propre égard.

En terme de régulation, le langage entre en particulier en jeu dans des processus de contretransfert. Ceux-ci sont constitutifs de la relation clinique et se rapportent aux réactions du soignant en présence du soigné - projections, ressentis, etc. -, réactions dont l'analyse peut permettre de mieux comprendre ce qui se joue pour le patient, en particulier dans le cadre des soins psychothérapeutiques. Ces réactions à l'autre peuvent ne pas être réfléchies de façon explicite (" moi je me sens très gros ", "j'ai l'impression de revêtir une sorte de blindage ", " il y a une résonance... c'est dans mon corps c'est tout mon corps qui réagit à ça »), mais le sont parfois très clairement, comme lorsqu'un psychothérapeute indique : "je me dis peutêtre que je m'identifie vraiment beaucoup à l'enfant et que je fais comme lui ».

Il a été montré de façon importante que le contre-transfert est aussi décisif que le transfert du patient dans la relation thérapeutique (Devereux, 1973). Il apparaît à la lumière de nos entretiens que les projections de type contre-transfert que le soignant développe à propos d'un patient ne sont pas limitées au cadre psychothérapeutique mais se manifestent chez une grande partie des soignants.

Nous pouvons mentionner enfin qu'il serait intéressant de disposer, pour une première rencontre soignant-soigné donnée, à la fois d'un entretien avec le soignant et d'un entretien avec le soigné. En effet, le second de ces entretiens nous permettrait d'appréhender les effets perlocutoires des interventions du soignant, et de vérifier si les intentions derrière ces messages se traduisent effectivement par les effets recherchés.

\section{Conclusion}

A partir d'une analyse générale de l'expérience subjective du soignant lors d'une première rencontre avec un patient, nous avons tenté d'analyser la place occupée par le langage dans le processus de soin. Nous avons considéré cette place dans le cadre d'une articulation entre le niveau microscopique d'une succession de phénomènes micro-expérientiels et le niveau macroscopique d'une activité finalisée visant le diagnostic et la création d'une alliance thérapeutique. Dans ce cadre, le langage du patient et celui du soignant jouent des rôles complémentaires pour le soignant: source d'information sur le patient, le langage entre également en jeu de façon interne et externe pour des actes d'évaluation, de régulation et d'intervention. Même si notre étude est centrée sur la première rencontre, il ne fait pas de doute que cette richesse se manifeste également ultérieurement dans le processus de soin.

L'intérêt d'une approche en première personne est de mettre clairement en lumière la diversité et la complexité de l'activité langagière. Le fait de pouvoir accéder à l'expérience intime du soignant offre une perspective fort différente de celle des études en troisième personne et à visée objective, et révèle en particulier le rôle invisible du langage, parallèlement à son rôle plus directement visible. Mieux prendre conscience de ce pan invisible de l'activité peut être au bénéfice des soignants et des soignés, et conduire à des relations plus authentiques, plus porteuses de sens et facilitatrices du processus de soin. 


\section{Références}

Ardito Rita B. et Rabellino Daniela. (2011). Therapeutic Alliance and Outcome of Psychotherapy: Historical Excursus, Measurements, and Prospects for Research. Frontiers in Psychology, vol 2, $\mathrm{n}^{\circ} 270$.

Austin John Langshaw. (1991). Quand dire, c'est faire (How to do Things with Words, 1962), Paris: Poche.

Barber Jacques P., Connolly Mary Beth, Crits-Christoph Paul, Gladis Lynn et Siqueland Lynne. (2000). Alliance predicts patients' outcome beyond in-treatment change in symptoms. Journal of Consulting and Clinical Psychology, vol. 68, n 6, p. 10271032.

Burloud Albert. (1927). La Pensée conceptuelle, essai de psychologie générale. Thèse principale pour le doctorat es-lettres, Université de Lyon.

Cuvelier Lucie. (2018). Never the first time on a patient: the stakes of high-fidelity simulation for safety training. Development and Learning in Organizations, vol. 32, n 5, p. $23-$ 25.

Daniellou François et Rabardel Pierre. (2005). Activity-oriented approaches to ergonomics: Some traditions and communities. Theoretical Issues in Ergonomics Science, vol. 6, $\mathrm{n}^{\circ}$ 5, p. 353-357.

De Sousa Santos Boaventura. (2018). The End of the Cognitive Empire: The Coming of Age of Epistemologies of the South, Duke University Press.

Dejours Christophe. (2007). Conjurer la violence : travail, violence et santé, Éditions Payot \& Rivages.

Denis Jennifer. (2016). Evaluation of Therapeutic Processes in Clinical Crisis Intervention. Experts' lived experiences of therapeutic action. Thèse de doctorat en Sciences Psychologiques et de l'Education, University de Mons.

Depraz Natalie. (2014). Première, deuxième, troisième personne, Zeta Books.

Depraz, Natalie, Varela Francisco et Vermersch Pierre. (2003). On becoming aware, John Benjamin.

Devereux Georges. (1973). Psychoanalysis and the Occult, International Universities Press.

Dieumegard Gilles, Nogry Sandra, Ollagnier-Beldame Magali et Perrin Nicolas. (2019). Lived experience as a unit of analysis for the study of learning. Learning, Culture, and Social Interaction, in press (published online, doi.org: 10.1016/j.lcsi.2019.100345)

Faingold Nadine. (1997). Contre-exemple et recadrage en analyse de pratiques. In Pierre Vermersch et Maryse Maurel (éds.), Pratiques de l'entretien d'explicitation, Paris : ESF.

Garfinkel Harold. (1967). Studies in Ethnomethodology, Prentice-Hall.

Gendlin Eugene T. (1997). Experiencing and the creation of meaning (1962), Northwestern University Press.

Giorgi Amedeo. (2009). The descriptive phenomenological method in psychology: A modified Husserlian approach, Duquesne University Press.

Good Byron. (1994). Medicine, Rationality, and Experience, Cambridge University Press.

Henry Julie. (2019). Commentary 2: "neither ill will nor a deliberate intention: what focus groups say about professional practices in hospitals". Journal of Empirical Research on Human Research Ethics, vol. 14, n 5, p. 498-500. 
Hurlburt Russell T. et Heavey Christopher L. (2006). Exploring inner experience: The Descriptive Experience Sampling method, John Benjamins.

Macewan Gregory H. (2008). The Efforts of Therapists in the First Session To Establish a Therapeutic Alliance. Master thesis in Psychology, University of Massachusetts Amherst.

McAllister Margaret M., Matarasso Beth J., Dixon, B. et Shepperd C. (2004). Conversation starters: re-examining and reconstructing first encounters within the therapeutic relationship. Journal of Psychiatric and Mental Health Nursing, vol. 11, p. 575-582.

Messer August. (1906). Experimentell-psychologische Untersuchungen über das Denken, Würzburg

Moscovici Serge. (2000). Social representations: Explorations in social psychology, Polity Press.

Nisbett Richard E. et Wilson Timothy D. (1977). Telling more than we can know: Verbal reports on mental processes. Psychological Review, vol. 84, n 3, p. 231-259.

Ollagnier-Beldame Magali et Cazemajou Anne. (2019). Intersubjectivity in first encounters between healthcare practitioners and patients: Micro-phenomenology as a way to study lived experience. The Humanistic Psychologist, vol. 47, n 4, p. 404-425.

Pauen, Michael. (2012). The Second-Person Perspective. Inquiry, vol. 55, n 1, p. 33-49.

Petitmengin Claire. (2001). L'expérience intuitive, L'Harmattan.

Petitmengin Claire. (2006). Describing one's subjective experience in the second person. An interview method for the science of consciousness. Phenomenology and the Cognitive Sciences, vol. 5, p. 229-269.

Petitmengin Claire, Remillieux Anne, Cahour Béatrice et Carter-Thomas Shirley. (2013). A gap in Nisbett and Wilson's findings? A first-person access to our cognitive processes. Consciousness and Cognition, vol. 22, n 2, p. 654-669.

Petitmengin Claire, Remillieux Anne et Valenzuela-Moguillansky Camila. (2019). Discovering the structures of lived experience. Towards a micro-phenomenological analysis method. Phenomenology and the Cognitive Sciences, vol. 18, p. 691-730.

Przyrembel Marisa et Singer Tania. (2018). Experiencing meditation - Evidence for differential effects of three contemplative mental practices in microphenomenological interviews. Consciousness and Cognition, vol. 62, p. 82-101.

Rabardel Pierre. (2005). Instrument, activité et développement du pouvoir d'agir. In Philippe Lorino et Régine Teulier (éds.), Entre connaissance et organisation: l'activité collective. La Découverte, p. 251-265.

Rogers Carl R. (1957). The necessary and sufficient conditions of therapeutic personality change. Journal of Consulting Psychology, vol. 21, n 2, p. 95-103.

Saldaña Johnny. (2011). Fundamentals of Qualitative Research (1st Edition), Oxford University Press.

Schacter Daniel. (2001). The seven sins of memory, Houghton Mifflin.

Shear Jonathan et Varela Francisco. (1999). The View from Within. First-person approaches to the study of consciousness, Imprint Academic.

Siksou Maryse. (2008). Georges Libman Engel (1913-1999). Le modèle biopsychosocial et la critique du réductionnisme biomédical. Le Journal Des Psychologues, vol. 260, p. $52-55$.

Stern Daniel N. (2004). The Present Moment In Psychotherapy and Everyday Life, W. W. Norton \& Company. 
Tronto Joan (2009). Un monde vulnérable, pour une politique du care (Moral Boundaries: a Political Argument for an Ethic of care, 1993), La Découverte.

Valenzuela-Moguillansky Camila et Vásquez-Rosati Alejandra. (2019). An Analysis Procedure for the Micro-Phenomenological Interview. Constructivist Foundations, vol. $14, \mathrm{n}^{\circ} 2$, p. 123-145.

Vargas-Schaffer Grisell et Cogan Jennifer. (2014). Patient therapeutic education: placing the patient at the centre of the WHO analgesic ladder. Canadian Family Physician, vol. $60, n^{\circ} 3$, p. $235-241$.

Vermersch Pierre. (1994). L'entretien d'explicitation en formation initiale et en formation continue, Editions Sociales Françaises.

Vermersch Pierre. (2000a). Approche du singulier. In Centre de recherche sur la formation du Conservatoire national des arts et métiers (éd.), L'Analyse de la singularité de l'action. Presses Universitaires de France, p. 239-266.

Vermersch Pierre. (2000b). Conscience directe et conscience réfléchie. Intellectica, vol. 2, $\mathrm{n}^{\circ} 31$, p. 269-311.

Vermersch Pierre. (2012). Explicitation et phénoménologie, Presses Universitaires de France.

\section{Remerciements}

Nous remercions Anne Cazemajou pour sa contribution à la collecte et l'analyse préliminaire des données. Nous remercions également l'ensemble des soignants qui ont accepté de s'entretenir avec nous sur l'une de leurs premières rencontres.

Ce travail a été possible grâce à un financement du projet ASLAN (ANR-10-LABX-0081) de l'Université de Lyon, dans le cadre du programme français « Investissements d'avenir » de l'Agence Nationale de la Recherche. 